\title{
Automated 3D trabecular bone structure analysis of the proximal femur-prediction of biomechanical strength by CT and DXA
}

\author{
T. Baum • J. Carballido-Gamio • M. B. Huber • D. Müller • R. Monetti • C. Räth • \\ F. Eckstein • E. M. Lochmüller • S. Majumdar • E. J. Rummeny • T. M. Link •
}

J. S. Bauer

Received: 21 May 2009 / Accepted: 1 October 2009 / Published online: 27 October 2009

(C) The Author(s) 2009. This article is published with open access at Springerlink.com

\begin{abstract}
Summary The standard diagnostic technique for assessing osteoporosis is dual X-ray absorptiometry (DXA) measuring bone mass parameters. In this study, a combination of DXA and trabecular structure parameters (acquired by computed tomography [CT]) most accurately predicted the biomechanical strength of the proximal femur and allowed for a better prediction than DXA alone.

Introduction An automated 3D segmentation algorithm was applied to determine specific structure parameters of the trabecular bone in CT images of the proximal femur. This was done to evaluate the ability of these parameters for predicting biomechanical femoral bone strength in comparison with bone mineral content (BMC) and bone mineral density (BMD) acquired by DXA as standard diagnostic technique.
\end{abstract}

T. Baum $(\bowtie) \cdot$ D. Müller • E. J. Rummeny • J. S. Bauer Institut für Röntgendiagnostik, Klinikum Rechts der Isar, Technische Universität München,

Ismaninger Str. 22,

81675 Munich, Germany

e-mail: thbaum@gmx.de

J. Carballido-Gamio - M. B. Huber · S. Majumdar · T. M. Link Musculoskeletal and Quantitative Imaging Research,

Department of Radiology, University of California, San Francisco, 185 Berry Street, Suite 350,

San Francisco, CA 94107, USA

R. Monetti · C. Räth

Max-Planck-Institut für Extraterrestrische Physik,

Giessenbachstr. 1,

85748 Garching, Germany

F. Eckstein • E. M. Lochmüller

Institute of Anatomy and Musculoskeletal Research,

Paracelsus Medical University Salzburg,

Strubergasse 21,

5020 Salzburg, Austria
Methods One hundred eighty-seven proximal femur specimens were harvested from formalin-fixed human cadavers. BMC and BMD were determined by DXA. Structure parameters of the trabecular bone (i.e., morphometry, fuzzy logic, Minkowski functionals, and the scaling index method [SIM]) were computed from CT images. Absolute femoral bone strength was assessed with a biomechanical sideimpact test measuring failure load (FL). Adjusted FL parameters for appraisal of relative bone strength were calculated by dividing FL by influencing variables such as body height, weight, or femoral head diameter.

Results The best single parameter predicting FL and adjusted FL parameters was apparent trabecular separation (morphometry) or DXA-derived BMC or BMD with correlations up to $r=0.802$. In combination with DXA, structure parameters (most notably the SIM and morphometry) added in linear regression models significant information in predicting FL and all adjusted FL parameters (up to $R_{\text {adj }}=0.872$ ) and allowed for a significant better prediction than DXA alone.

Conclusion A combination of bone mass (DXA) and structure parameters of the trabecular bone (linear and nonlinear, global and local) most accurately predicted absolute and relative femoral bone strength.

Keywords Computed tomography · Failure load . Osteoporosis · Proximal femur . Trabecular bone structure

\section{Introduction}

Osteoporotic hip fractures are associated with an increased mortality and a reduced quality of life [1,2]. The standard diagnostic technique for assessing osteoporosis and monitoring therapy is dual X-ray absorptiometry (DXA) measuring 
bone mineral density (BMD) [3]. BMD can predict femoral bone strength and fracture risk to some extent, but BMD values of patients with and without femur fractures overlap [4-9]. BMD does not encompass bone quality, but bone quality is, in addition to bone density, a substantial parameter for predicting bone strength. Bone quality can be partly assessed by analyzing trabecular architecture. For this reason, trabecular bone structure analysis is an important research topic. Imaging modalities to characterize trabecular bone structure include computed tomography (CT) and magnetic resonance imaging (MRI) [10]. Although the typical spatial resolutions of clinical CT and MRI scanners are not high enough to depict single trabeculae, several studies showed high correlations between trabecular bone structure parameters obtained from CT or MRI and those determined in highresolution radiographs or micro-CT images as standard of reference $[11,12]$. Therefore, $\mathrm{CT}$ and MRI are adequate techniques to analyze trabecular bone structure, even though large errors remain for in vivo application. A multitude of trabecular bone structure parameters have been developed during the last years. Morphometric parameters such as bone fraction $(\mathrm{BF})$, trabecular number $(\mathrm{TbN})$, trabecular separation (TbSp), and trabecular thickness ( $\mathrm{TbTh}$ ) were frequently used and showed significant correlations with the mechanical properties of the femoral bone in multiple studies [13-15]. More sophisticated parameters based on fuzzy logic and scaling index method (SIM) as well as Minkowski functionals (MF) have been designed recently to characterize trabecular bone structure [16-21]. However, all of the abovementioned parameters have never been compared simultaneously in a single study among themselves and with bone mineral content (BMC) and BMD measured by DXA as standard diagnostic technique with regard to their predictive capability of femoral bone strength. Additionally, standardized, automated locations are required for good reproducibility of the trabecular bone structure parameters, since the proximal femur is very heterogeneous [22, 23].

Therefore, the first objective of this in vitro study was to use an automated 3D segmentation algorithm to determine specific structure parameters of the trabecular bone in CT images of the proximal femur, specifically morphometry, fuzzy logic, MF, and SIM. The second objective then was to test the hypothesis that these parameters could significantly improve the prediction of absolute and relative femoral bone strength beyond bone mass alone, as measured by DXA.

\section{Material and methods}

Femur specimens

Femur specimens were harvested from 248 formalin-fixed human cadavers. The donors had dedicated their body for educational and research purposes to the Institute of Anatomy in Munich prior to death, in compliance with local institutional and legislative requirements. Aside from osteoporosis, all pathological bone changes like bone metastases, hematological, or metabolic bone disorders were exclusion criteria for the study. Therefore, biopsies were taken from the iliac crest of all donors and examined histologically. Furthermore, radiographs were obtained from all specimens. If fractures, osteolytic changes, or other focal abnormalities were detected in the images, the respective donor was excluded from the study. Femur specimens that fractured during preparation or had distal shaft fractures in the biomechanical testing were also excluded. Using these criteria, 187 donors were included in the study, 93 females and 94 males. The donors had a mean age \pm standard deviation (SD) of $79 \pm 10$ years (range $52-100$ years). The body height (BH) and body weight (BW) of each donor were measured. Surrounding soft tissue was completely removed from the femora and femoral head and neck diameter were measured. The head diameter was defined as the largest diameter of the femoral head in a plane orthogonal to the femoral neck axis. The neck diameter was the smallest diameter of the neck in a plane orthogonal to the femoral neck axis. For the purpose of conservation, all specimens were stored in formalin solution during the study. The specimens were degassed at least $24 \mathrm{~h}$ before imaging to prevent air artifacts.

\section{DXA measurements}

DXA was used to determine BMC and BMD in four regions of interest (ROIs) in each femur specimen. These ROIs were the neck ROI, greater trochanter ROI, intertrochanteric ROI, and consisting of the three ROIs, the total proximal femur ROI. DXA measurements were performed with a Prodigy Scanner (GE/Lunar; GE Medical Systems, Milwaukee, WI, USA). The femur specimens were positioned similar to in vivo examination conditions: mildly internally rotated in a vessel filled with tap water up to $15 \mathrm{~cm}$ in height to simulate soft tissue. The measurements were evaluated by using the Lunar Prodigy Encore 2002 software (GE Medical Systems). The software was additionally used to assess femoral neck length (FNL) of each specimen.

\section{CT imaging}

CT images of the proximal femora were acquired for the structure analysis of the trabecular bone by using a 16-row CT scanner (Sensation 16; Siemens Medical Solutions, Erlangen, Germany). The specimens were placed in plastic bags filled with $4 \%$ formalin-water solution. The plastic bags were sealed after air was removed by a vacuum pump. These bags were positioned in the scanner with mild 
internal rotation of the femur to simulate the conditions as in an in vivo examination of the pelvis and proximal femur. Three specimens were scanned twice with repositioning to determine reproducibility. The applied scan protocol had a collimation and a table feed of $0.75 \mathrm{~mm}$ and a reconstruction index of $0.5 \mathrm{~mm}$. Further scanning parameters were $120 \mathrm{kVp}, 100 \mathrm{~mA}$, an image matrix of $512 \times 512$ pixels, and a field of view of $100 \mathrm{~mm}$. From a high-resolution reconstruction algorithm (kernel $\mathrm{U} 70 \mathrm{u}$ ) resulted an inplane spatial resolution of $0.29 \times 0.29 \mathrm{~mm}^{2}$, determined at $\rho=10 \%$ of the modulation transfer function. Voxel size was $0.19 \times 0.19 \times 0.5 \mathrm{~mm}^{3}$. For calibration purposes, a reference phantom with a bone-like and a water-like phase (Osteo Phantom, Siemens Medical Solutions) was placed in the scanner below the specimens.

\section{CT image processing}

Three volumes of interest (VOIs) were fitted automatically in the trabecular part of the femoral head, neck, and greater trochanter. The algorithm was described in detail by Huber et al. for trabecular BMD analysis [24]. The outer surface of the cortical shell of the femur was segmented automatically by a threshold-based technique. The segmentation had to be corrected manually in 14 out of 187 cases due to thin cortical shell. Causes were focal bone loss due to advanced osteoporosis or adjacent anatomic structures, such as blood vessels, penetrating the cortex. After completed segmentation, an ellipsoid VOI was automatically fitted in the femoral head as well as a cylindric VOI in the femoral neck and an irregular VOI in the greater trochanter (Fig. 1).

To obtain the head VOI, an ellipse was fitted to the superior bone surface points of the femoral head using a Gaussian-Newton least squares technique. The fitted ellipse was scaled down to $75 \%$ of its original size to account for cortical bone and shape irregularities of the femoral head and saved as head VOI.

For the cylindric neck VOI, an initial axis of the cylinder was established between the center of mass of the fitted ellipse and the intersection between the prolonged neck axis and the lateral bone surface. Based on this initial axis and the bone surface points of the neck, a first cylinder was fitted in the neck using a Gaussian-Newton least squares technique. The axis of the first cylinder was retained unchanged for the final cylinder. To account for cortical bone and shape irregularities, final cylinder length was defined as $65 \%$ of the radius of the first cylinder. The radius of the final cylinder was hereupon optimized by using the bone surface points of the neck. The final cylinder was saved as neck VOI.

To define the trochanteric VOI, the cylinder axis was prolonged as far as the intersection with the lateral bone surface. Based on the relative position of the bone surface points to this intersection and the cylinder axis, surface regions corresponding to the trochanter, inferior part of the neck, and superior part of the shaft were determined. The surface region of the trochanter was used to fit a cone in the trochanter using a Gaussian-Newton least squares

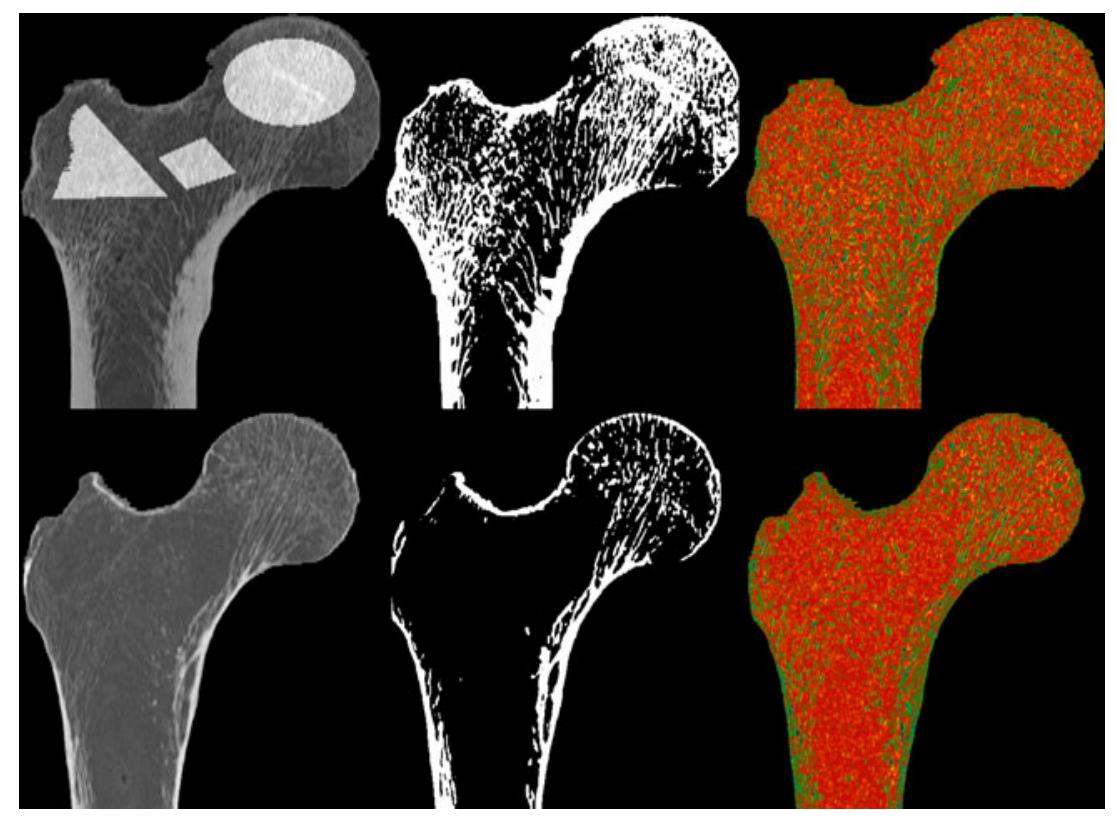

Fig. 1 Comparison of a healthy (upper row) and an osteoporotic femur (lower row): 3D visualization of the fitted VOIs: head (ellipsoid), neck (cylinder), and trochanter (irregular) in the original
CT data (left), binarized dataset according to $V_{\mathrm{MF}}$ (middle) and colorcoded $m_{P(\alpha)}$-map (right) 
technique. The cone was discarded, but the relative position of the bone points to the fitted cone axis and the cylinder axis was assessed. According to their relative position, they were labeled as "trochanteric" or "nontrochanteric" bone points. The trochanteric bone points were saved as trochanteric VOI.

All image-processing steps were conducted at Sun Workstations (Sun Microsystems, Santa Clara, CA, USA) with custom-built software based on MATLAB (Version 7.0, The MathWorks, Natick, MA, USA).

\section{Trabecular structure analysis}

The following structure parameters of the trabecular bone were determined in the fitted VOIs:

Morphometry Binarization of the $\mathrm{CT}$ images was required to calculate 2D morphometric parameters. For this purpose, we applied a previously optimized global threshold which was determined to be $200 \mathrm{mg} / \mathrm{cm}^{3}$ hydroxyapatite [13]. After binarization, four morphometric parameters were calculated in analogy to standard histomorphometry using the mean intercept length method [25]: BF (resulting from bone volume divided by total volume), $\mathrm{TbN}, \mathrm{TbSp}$, and $\mathrm{TbTh}$. Parameters were labeled as apparent (app.) values, since, given the limited spatial resolution, they cannot depict the true trabecular structure.

Fuzzy logic Previously, fuzzy logic was applied on magnetic resonance images to characterize trabecular bone structure $[19,21,26]$. The application on our CT images was conducted similarly. For the calculation of the 3D fuzzy logic parameters, no binarization was required. In a first step, which is known as "concentration," each voxel within a VOI was multiplied by itself to increase contrast. Then each voxel was fuzzily segmented into the bone subset and the marrow subset by using fuzzy c-means clustering. Voxels were allowed partial memberships in both subsets at the same time. The membership value of the voxel in the bone subset was considered as the amount of bone in the voxel, since the range of values for each voxel was from 0 to 1 , where 0 represented a marrow voxel, 1 represented a bone voxel, and any value in between represented the corresponding $\mathrm{BF}$ of that voxel. Thus, fuzzy-bone volume fraction (f-BVF) maps could be generated. Based on these f-BVF maps, the fuzzy-bone fraction (f-BF) of the VOI could be calculated. Furthermore, 3D linear and quadratic indices of fuzziness and 3D logarithmic and exponential fuzzy entropies were computed according to Carballido-Gamio et al. [19].

SIM-derived parameter The SIM is a tool for the structural characterization of arbitrary-dimensional point distribu- tions. For trabecular bone structure analysis, tomographic images can be interpreted as four-dimensional point distributions where each point (voxel) is defined by its $x$-, $y$-, and $z$-coordinate and its intensity value. A binarization of the images is not necessary. The 3D-based scaling index $\alpha$ can be calculated for each point of the distribution; $\alpha$ reveals the local dimensionality: rod-like structures $(\alpha \sim 1)$, plate-like structures $(\alpha \sim 2)$, and random background $(\alpha \sim 3)$ can be differentiated. Nonlinear texture parameters can be derived from the probability distributions $P(\alpha)$ of the scaling indices $\alpha$. According to previous studies, we extracted the scaling indices $\alpha$ in our CT images and calculated $m_{P(\alpha)}$ with two sliding windows in the $P(\alpha)$ spectrum [18, 20] (Fig. 1). The position and width of the two windows were chosen to achieve optimal correlations between $m_{P(\alpha)}$ and failure load (FL).

Minkowski functionals The MF can be applied to multidimensional objects to characterize the composition of their components. In 3D, the four MFs, namely, volume $\left(V_{\mathrm{MF}}\right)$, surface area $\left(\operatorname{Sur}_{\mathrm{MF}}\right)$, mean integral curvature $\left(\mathrm{Curv}_{\mathrm{MF}}\right)$, and Euler characteristic $\left(\mathrm{Eul}_{\mathrm{MF}}\right)$, entirely characterize one object. MF are nonlinear topological parameters and were calculated for all thresholds between 180 and $220 \mathrm{mg} / \mathrm{cm}^{3}$ hydroxyapatite in steps of $0.1 \mathrm{mg} / \mathrm{cm}^{3}$ over all three VOIs of each specimen, according to the algorithm of Michielsen et al. [27] (Fig. 1). Further statistical analysis was conducted only with the optimal threshold for each MF that achieved the highest correlation with FL $\left(201.0 \mathrm{mg} / \mathrm{cm}^{3}\right.$ for $V_{\mathrm{MF}}$, $203.8 \mathrm{mg} / \mathrm{cm}^{3}$ for $\operatorname{Sur}_{\mathrm{MF}}, 208.6 \mathrm{mg} / \mathrm{cm}^{3}$ for Curv $\mathrm{CF}$, and $196.2 \mathrm{mg} / \mathrm{cm}^{3}$ for $\mathrm{Eul}_{\mathrm{MF}}$ ).

Structure analysis was performed with custom-built software based on Interactive Data Language (IDL, Research Systems, Boulder, CO, USA).

\section{Biomechanical femoral bone strength}

Absolute femoral bone strength was assessed with a biomechanical side-impact test measuring FL, described in detail previously [28]. In brief, a lateral fall on the greater trochanter was simulated. Femoral head and shaft were faced downward and could be moved independently from each other while the load was applied on the greater trochanter by using a universal testing machine (Zwick 1445; Zwick, Ulm, Germany) with a $10-\mathrm{kN}$ force sensor and dedicated software. FL was defined as the peak of the load-deformation curve. Since FL depends on influencing variables such as bone size, relative femoral bone strength had to be appraised for better interpretation of the clinical utility. For appraisal of the relative bone strength, FL was adjusted to age, BH, BW, femoral head diameter (HD), femoral neck diameter (ND), and FNL. For this purpose, 
FL was divided by the respective parameter, whereby six adjusted FL parameters were generated.

\section{Statistical analysis}

Mean values, SDs, and coefficients of variations (CVs) of all parameters were calculated for all specimens. The Kolmogorov-Smirnov test showed for the vast majority of parameters significant differences from a normal distribution. Therefore, differences between ROIs or VOIs were evaluated with the Mann-Whitney $U$ test considering the Bonferroni correction for multiple comparisons. Correlations between two parameters were evaluated with the Spearman correlation coefficient $(r)$. Significant differences between correlation coefficients were assessed using the Fisher $Z$ transformation. Since normal distribution could be assumed for FL and the six adjusted FL parameters, multiple linear regression analysis was performed to assess if the structure parameters and the best DXA parameter (BMC or BMD) could significantly better predict FL, respectively, of each of the adjusted FL parameters, compared to the best DXA parameter alone. Structure parameters were included in the regression models if the level of significance was $p<0.05$. Adjusted regression coefficients $\left(R_{\text {adj }}\right)$ were calculated for each model. Models were compared using the extra sum-of-squares $F$ test.

The statistical analyses were performed with SPSS (SPSS, Chicago, IL, USA) and supervised by a statistician. All tests were done using a two-sided 0.05 level of significance.

\section{Reproducibility}

Reproducibility errors were calculated for the morphometry measures. For this purpose, the automatic segmentation for six randomly chosen specimens (three females and three males) was checked by two investigators (T.B. and M.B.H.) independently of each other and manually corrected, if required. The reproducibility errors were calculated in absolute numbers as root mean square average of the errors of each specimen and on percentage basis as the root mean square average of the single CV per specimen [29]. Furthermore, three specimens were scanned twice with repositioning. Segmentation and VOI-fitting algorithm was applied on both acquisitions. As described above, segmentation was controlled and reproducibility errors were calculated.

\section{Results}

Average BMD measured using DXA was significantly lower in the trochanter ROI $\left(0.67 \mathrm{~g} / \mathrm{cm}^{2}\right)$ and neck ROI $\left(0.71 \mathrm{~g} / \mathrm{cm}^{2}\right)$ compared to the intertrochanteric ROI $\left(0.96 \mathrm{~g} / \mathrm{cm}^{2}\right)$ and total proximal femur ROI $\left(0.80 \mathrm{~g} / \mathrm{cm}^{2} ; p<0.05\right.$; Table 1). All morphometric parameters showed significant differences between head, neck, and trochanter $(p<0.05)$. App.BF, app. $\mathrm{TbN}$, and app.TbTh were highest in the head and lowest in the neck. A converse result was found for app.TbSp (Table 1). Highest values for each fuzzy logic parameter and SIMderived $m_{P(\alpha)}$ were obtained in the head and lowest values in the neck (Table 1).

Reproducibility errors of the morphometric parameters amounted to $0.11-9.41 \%$ for segmentation and 1.59 $33.81 \%$ for segmentation with repositioning (Table 2). Reproducibility errors were lowest for app.BF in the head and highest for app.TbSp in the neck.

Correlation coefficients of FL and all adjusted FL parameters with BMC, BMD, and trabecular structure parameters are listed in Table 3, except for FL/ND and FL/FNL, since correlation coefficients of FL/HD, FL/ND, and FL/FNL had comparable values.

$\mathrm{BMC}$ of the total proximal femur (total BMC) showed the highest correlation with FL $(r=0.802$; Fig. 2). By adjusting FL to $\mathrm{BH}$ and age, differences between highest $\mathrm{BMC}$ and highest BMD correlation coefficients decreased $(\Delta r=0.015$ and $\Delta r=0.008$, respectively; Table 3). After adjustment of FL to BW and measures of femoral bone size, highest correlations were observed for BMD and not for BMC. The highest correlation coefficient of FL and all adjusted FL parameters with BMC or BMD did not significantly differ from the highest of the trabecular structure parameters $(p>0.05)$.

App.TbSp in the femoral head showed the highest correlation of all morphometric parameters with FL and all adjusted FL parameters (up to $r=-0.743$ for FL/HD; Fig. 2). By adjusting FL to BH and measures of femoral bone size, higher correlation coefficients were achieved for app.TbSp in the head (Table 3). Correlation of FL/HD with app.TbSp in the head was even higher than those with $\mathrm{BMC}$ and BMD.

After adjustment of $\mathrm{FL}$ to $\mathrm{BH}$, measures of femoral bone size and age, correlation coefficients of fuzzy logic parameters and SIM-derived $m_{P(\alpha)}$ remained almost unchanged (Table 3). Fuzzy logic parameters and $m_{P(\alpha)}$ had lower correlations with FL and all adjusted FL parameters than the morphometric parameters. Highest correlations were observed for $\mathrm{f}-\mathrm{BF}$ in the head (up to $r=0.506$ for FL/ HD; Fig. 2) and for the neck $m_{P(\alpha)}$ with FL/HD $(r=0.493$; Fig. 2).

The highest correlation of all MF with FL was found for $V_{\mathrm{MF}}(r=0.744$; Fig. 2). Adjusted FL parameters showed lower correlations with MF (Table 3), but the respective highest correlation coefficient did not significantly differ from the overall highest correlation coefficient achieved by BMC, BMD, or app.TbSp in the head $(p>0.05)$. 
Table 1 Mean values, SDs, and $\mathrm{CVs}$ of investigated parameters

\begin{tabular}{|c|c|c|c|c|}
\hline Parameter & Region & mean & SD & $\mathrm{CV}$ \\
\hline Age [years] & & 79.3 & 10.1 & 0.127 \\
\hline $\mathrm{BH}[\mathrm{cm}]$ & & 165 & 9 & 0.055 \\
\hline BW [kg] & & 59.5 & 15.0 & 0.252 \\
\hline Head diameter [mm] & & 49.1 & 4.1 & 0.084 \\
\hline Neck diameter $[\mathrm{mm}]$ & & 27.8 & 3.2 & 0.115 \\
\hline $\mathrm{FNL}[\mathrm{mm}]$ & & 98.1 & 8.3 & 0.082 \\
\hline $\mathrm{FL}[\mathrm{N}]$ & & 4,008 & 1,518 & 0.379 \\
\hline \multirow[t]{4}{*}{$\mathrm{BMC}[\mathrm{g}]$} & Neck & 3.84 & 1.15 & 0.300 \\
\hline & Trochanter & 10.08 & 3.81 & 0.378 \\
\hline & Intertrochanteric & 14.49 & 3.92 & 0.271 \\
\hline & Total & 28.35 & 8.30 & 0.293 \\
\hline \multirow[t]{4}{*}{$\mathrm{BMD}\left[\mathrm{g} / \mathrm{cm}^{2}\right]$} & Neck & 0.71 & 0.18 & 0.254 \\
\hline & Trochanter & 0.67 & 0.18 & 0.269 \\
\hline & Intertrochanteric & 0.96 & 0.23 & 0.240 \\
\hline & Total & 0.80 & 0.19 & 0.238 \\
\hline app.BF & Head & 0.55 & 0.14 & 0.255 \\
\hline app.TbN $\left[\mathrm{mm}^{-1}\right]$ & & 0.73 & 0.11 & 0.151 \\
\hline app.TbSp [mm] & & 0.66 & 0.51 & 0.773 \\
\hline app.TbTh [mm] & & 0.79 & 0.31 & 0.392 \\
\hline app.BF & Neck & 0.10 & 0.09 & 0.900 \\
\hline app.TbN $\left[\mathrm{mm}^{-1}\right]$ & & 0.27 & 0.21 & 0.778 \\
\hline app.TbSp [mm] & & 11.20 & 12.09 & 1.079 \\
\hline app.TbTh [mm] & & 0.29 & 0.08 & 0.276 \\
\hline app.BF & Trochanter & 0.15 & 0.10 & 0.667 \\
\hline app.TbN $\left[\mathrm{mm}^{-1}\right]$ & & 0.39 & 0.20 & 0.513 \\
\hline app.TbSp [mm] & & 5.92 & 10.09 & 1.740 \\
\hline app.TbTh [mm] & & 0.35 & 0.09 & 0.257 \\
\hline $\mathrm{f}-\mathrm{BF}$ & Head & 0.442 & 0.033 & 0.075 \\
\hline lin.fuzziness & & 0.349 & 0.011 & 0.032 \\
\hline log.entropy & & 0.572 & 0.013 & 0.023 \\
\hline $\mathrm{f}-\mathrm{BF}$ & Neck & 0.363 & 0.078 & 0.215 \\
\hline lin.fuzziness & & 0.326 & 0.034 & 0.104 \\
\hline log.entropy & & 0.544 & 0.041 & 0.075 \\
\hline $\mathrm{f}-\mathrm{BF}$ & Trochanter & 0.410 & 0.039 & 0.095 \\
\hline lin.fuzziness & & 0.344 & 0.013 & 0.038 \\
\hline log.entropy & & 0.565 & 0.016 & 0.028 \\
\hline \multirow[t]{3}{*}{$m_{P(\alpha)}$} & Head & 8.535 & 0.075 & 0.009 \\
\hline & Neck & 1.199 & 0.021 & 0.018 \\
\hline & Trochanter & 2.329 & 0.016 & 0.007 \\
\hline$V_{\mathrm{MF}}$ & Total & 374,633 & 166,163 & 0.444 \\
\hline $\operatorname{Sur}_{M F}$ & & 321,978 & 141,623 & 0.440 \\
\hline $\operatorname{Curv}_{\mathrm{MF}}$ & & $7,804.10$ & $4,332.32$ & 0.555 \\
\hline $\mathrm{Eul}_{\mathrm{MF}}$ & & 327.34 & $1,497.89$ & 4.576 \\
\hline
\end{tabular}

The best DXA and best multiple regression models for FL and all adjusted FL parameters are listed in Table 4. Structure parameters of the trabecular bone could add significant information in the multiple regression models.
The best multiple regression model for FL and each adjusted FL parameter showed significantly higher $R_{\mathrm{adj}}$ than the respective model of the best DXA parameter alone $(p<0.05)$. 
Table 2 Reproducibility errors of morphometric parameters on percentage basis and in absolute numbers

\begin{tabular}{|c|c|c|c|c|c|c|c|c|}
\hline & \multicolumn{2}{|l|}{ app.BF } & \multicolumn{2}{|c|}{ app.TbN } & \multicolumn{2}{|c|}{ app.TbSp } & \multicolumn{2}{|c|}{ app.TbTh } \\
\hline & & & $\%$ & $\mathrm{~mm}^{-1}$ & $\%$ & $\mathrm{~mm}$ & $\%$ & $\mathrm{~mm}$ \\
\hline \multicolumn{9}{|c|}{ Reproducibility errors for segmentation } \\
\hline Head & $0.11 \%$ & 0.0005 & 0.13 & 0.0010 & 0.27 & 0.0022 & 0.13 & 0.0013 \\
\hline Neck & $1.56 \%$ & 0.0022 & 0.99 & 0.0037 & 9.41 & 0.2582 & 1.63 & 0.0060 \\
\hline Trochanter & $0.66 \%$ & 0.0017 & 0.34 & 0.0015 & 0.15 & 0.0064 & 0.98 & 0.0045 \\
\hline \multicolumn{9}{|c|}{ Reproducibility errors for segmentation with repositioning } \\
\hline Head & $1.59 \%$ & 0.0095 & 5.00 & 0.0330 & 2.58 & 0.0141 & 6.18 & 0.0709 \\
\hline Neck & $5.68 \%$ & 0.0172 & 6.00 & 0.0312 & 33.81 & 0.9644 & 2.79 & 0.0137 \\
\hline Trochanter & $4.78 \%$ & 0.0134 & 4.65 & 0.0245 & 8.03 & 0.1653 & 5.08 & 0.0235 \\
\hline
\end{tabular}

\section{Discussion}

To the best of our knowledge, this was the first study to combine density information with morphometry, fuzzy logic, MF, and SIM for the prediction of femoral bone strength. DXA-derived BMC showed the highest correlation with FL, since both are strongly dependent on bone size. Therefore, relative femoral bone strength was appraised by adjusting FL to anthropometric factors. Thus, a gold standard was obtained, closely related to the clinically relevant fracture risk. In contrast to FL, relative bone strength showed lower differences between the highest correlation coefficients of BMC, BMD, and trabecular structure parameters. In combination with DXA, trabecular structure parameters (most notably the SIM and morphometry) added significant information in predicting FL and relative bone strength and allowed for a significantly better prediction than DXA alone.

Previous studies correlated morphometric parameters and BMD with FL obtained from whole-femur specimens by whole-body $\mathrm{CT}$ and MR, respectively $[13,14]$. In those studies, BMC and BMD yielded highest correlations with FL. Correlation coefficients for morphometric parameters versus FL were reported up to $r=0.69$ in case of MRI and up to $r=0.68$ in CT images, values comparable to our study. While Bauer et al. could not significantly improve correlation of BMC versus FL using additional morphometric parameters obtained by CT, this study demonstrated that a significant improvement is possible using morphometric, fuzzy logic, and nonlinear parameters.

MF and SIM-derived $m_{P(\alpha)}$ are those nonlinear structure parameters computed in this study. MF showed higher correlations with FL and adjusted FL parameter than $m_{P(\alpha)}$. One possible reason could be the calculation of MF over all three VOIs, resulting in higher information content. Using a sliding windows algorithm for MF parameter calculation, even higher correlations of MF versus FL (up to $r=0.91$ ) were reported in previous studies [16, 17]. $m_{P(\alpha)}$ was the only structure parameter used in this study revealing no global information but local information about the trabecular bone structure. It showed moderate correlations with FL and adjusted FL parameters, but provided additional information for predicting those pointed out in the multiple regression models. Boehm et al. extracted a different SIMderived parameter from MR images of femoral bone cubes and obtained a higher correlation with FL $(r=0.78)$ than this study [18]. Like the 3D digital topological analysis described by Wehrli et al., SIM and MF are further approaches to characterize 3D trabecular bone architecture $[30,31]$.

Fuzzy logic has not been applied to CT images for trabecular bone structure analysis. Patel et al. calculated fuzzy logic parameters in MR images of calcaneus specimens and reported nonsignificant correlations between fuzzy logic parameters and femoral FL [21]. In this study, significant correlations were obtained, but correlations were still lower than those of morphometric parameters. However, fuzzy logic could partly add information in the multiple regression models to predict FL and adjusted FL parameters.

We found correlation coefficients up to $r=0.802$ for BMC versus FL. These findings are consistent with previous studies [5, 32, 33]. It was not surprising that BMC showed the highest correlation with FL, since both are strongly dependent on bone size, in contrast to BMD and trabecular structure parameters. For in vivo fracture prediction, relative femoral bone strength is relevant, considering influencing variables such as anthropometric factors (BH, measures of femoral bone size, etc.) or age. Therefore, relative femoral bone strength was appraised by adjusting FL to those influencing variables. As an indication for adequate adjustment of FL to BH and femoral bone size, difference between highest BMC and highest BMD correlation coefficient decreased $(\Delta r=0.015)$, respectively; 
Table 3 Spearman correlation coefficients $\mathrm{r}$ of investigated parameters versus FL and adjusted FL

\begin{tabular}{|c|c|c|c|c|c|c|}
\hline Parameter & Region & Versus FL & Versus FL/BH & Versus FL/BW & Versus FL/HD & Versus FL/age \\
\hline Age [years] & & $-0.272 * *$ & $-0.262 * *$ & n.s. & $-0.274 * *$ & $-0.518^{* *}$ \\
\hline $\mathrm{BH}[\mathrm{cm}]$ & & $0.552 * *$ & $0.447 * *$ & $0.208^{*}$ & $0.299 * *$ & $0.532 * *$ \\
\hline BW [kg] & & $0.583^{* *}$ & $0.554 * *$ & n.s. & $0.513^{* *}$ & $0.592 * *$ \\
\hline $\mathrm{HD}$ [mm] & & $0.420 * *$ & $0.349 * *$ & $0.208^{*}$ & $0.196 * *$ & $0.384 * *$ \\
\hline \multirow[t]{4}{*}{$\mathrm{BMC}[\mathrm{g}]$} & Neck & $0.793^{* *}$ & $0.755^{* *}$ & $0.441^{* *}$ & $0.693^{* *}$ & $0.772 * *$ \\
\hline & Trochanter & $0.735^{* *}$ & $0.689 * *$ & $0.442 * *$ & $0.606^{* *}$ & $0.668 * *$ \\
\hline & Intertrochanteric & $0.776^{* *}$ & $0.750^{* *}$ & $0.467 * *$ & $0.693 * *$ & $0.764 * *$ \\
\hline & Total & $0.802 * *$ & $0.764 * *$ & $0.466^{* *}$ & $0.683 * *$ & $0.763^{* *}$ \\
\hline \multirow[t]{4}{*}{$\mathrm{BMD}\left[\mathrm{g} / \mathrm{cm}^{2}\right]$} & Neck & $0.766^{* *}$ & $0.749^{* *}$ & $0.445^{* *}$ & $0.717^{* *}$ & $0.764^{* *}$ \\
\hline & Trochanter & $0.763 * *$ & $0.734 * *$ & $0.425^{* *}$ & $0.669^{* *}$ & $0.723^{* *}$ \\
\hline & Intertrochanteric & $0.737 * *$ & $0.730 * *$ & $0.482 * *$ & $0.686^{* *}$ & $0.742 * *$ \\
\hline & Total & $0.766^{* *}$ & $0.749 * *$ & 0.460 & $0.707 * *$ & $0.755^{* *}$ \\
\hline app.BF & Head & $0.666^{* *}$ & $0.666^{* *}$ & $0.388 * *$ & $0.683 * *$ & $0.664 * *$ \\
\hline app.TbN $\left[\mathrm{mm}^{-1}\right]$ & & n.s. & n.s. & $0.173 *$ & n.s. & n.s. \\
\hline app.TbSp [mm] & & $-0.715^{* *}$ & $-0.726^{* *}$ & $-0.441 * *$ & $-0.743 * *$ & $-0.702 * *$ \\
\hline app.TbTh [mm] & & $0.540 * *$ & $0.529 * *$ & $0.292 * *$ & $0.513 * *$ & $0.551 * *$ \\
\hline app.BF & Neck & $0.565^{* *}$ & $0.562 * *$ & $0.352 * *$ & $0.576^{* *}$ & $0.584 * *$ \\
\hline app.TbN $\left[\mathrm{mm}^{-1}\right]$ & & $0.565^{* *}$ & $0.562 * *$ & $0.351^{* *}$ & $0.572 * *$ & $0.579^{* *}$ \\
\hline app.TbSp [mm] & & $-0.497 * *$ & $-0.489 * *$ & $-0.289 * *$ & $-0.513 * *$ & $-0.517 * *$ \\
\hline app.TbTh [mm] & & $0.508^{* *}$ & $0.508^{* *}$ & $0.319^{* *}$ & $0.517 * *$ & $0.534 * *$ \\
\hline app.BF & Trochanter & $0.567 * *$ & $0.538 * *$ & $0.288 * *$ & $0.470 * *$ & $0.502 * *$ \\
\hline app.TbN $\left[\mathrm{mm}^{-1}\right]$ & & $0.586^{* *}$ & $0.559 * *$ & $0.321 * *$ & $0.506^{* *}$ & $0.527 * *$ \\
\hline app.TbSp [mm] & & $-0.583 * *$ & $-0.555^{* *}$ & $-0.307^{* *}$ & $-0.510^{* *}$ & $-0.531 * *$ \\
\hline app.TbTh [mm] & & $0.428 * *$ & $0.401 * *$ & $0.161^{*}$ & $0.342 * *$ & $0.352 * *$ \\
\hline $\mathrm{f}-\mathrm{BF}$ & Head & $0.476^{* *}$ & $0.473 * *$ & $0.271 * *$ & $0.506^{* *}$ & $0.455^{* *}$ \\
\hline lin.fuzziness & & $0.350 * *$ & $0.350 * *$ & $0.233 * *$ & $0.417 * *$ & $0.344 * *$ \\
\hline qua.fuzziness & & $0.330 * *$ & $0.331 * *$ & $0.226^{* *}$ & $0.397 * *$ & $0.324^{*}$ \\
\hline log.entropy & & $0.368 * *$ & $0.368 * *$ & $0.239 * *$ & $0.436^{* *}$ & $0.361^{* *}$ \\
\hline exp.entropy & & $0.363 * *$ & $0.363 * *$ & $0.237 * *$ & $0.430 * *$ & $0.357 * *$ \\
\hline f-BF & Neck & $0.149 *$ & n.s. & n.s. & $0.207 *$ & $0,163^{*}$ \\
\hline f-BF & Trochanter & $0.332 * *$ & $0.300 * *$ & $0.175^{*}$ & $0.299 * *$ & $0.312 * *$ \\
\hline lin.fuzziness & & $0.366^{* *}$ & $0.334 * *$ & $0.173^{*}$ & $0.388 * *$ & $0.377 * *$ \\
\hline qua.fuzziness & & $0.355^{* *}$ & $0.323 * *$ & $0.163^{*}$ & $0.389 * *$ & $0.370^{* *}$ \\
\hline log.entropy & & $0.372 * *$ & $0.340 * *$ & $0.182 *$ & $0.385^{* *}$ & $0.379 * *$ \\
\hline exp.entropy & & $0.372 * *$ & $0.340 * *$ & $0.180 *$ & $0.389 * *$ & $0.380 * *$ \\
\hline \multirow[t]{3}{*}{$m_{P(\alpha)}$} & Head & $0.456^{* *}$ & $0.453 * *$ & $0.332 * *$ & $0.461 * *$ & $0.409 * *$ \\
\hline & Neck & $0.423 * *$ & $0.417 * *$ & $0.279 * *$ & $0.493 * *$ & $0.420 * *$ \\
\hline & Trochanter & $0.488 * *$ & $0.462 * *$ & $0.245^{* *}$ & $0.456 * *$ & $0.462 * *$ \\
\hline$V_{\mathrm{MF}}$ & Total & $0.744 * *$ & $0.700 * *$ & $0.427 * *$ & $0.581 * *$ & $0.717 * *$ \\
\hline $\operatorname{Sur}_{M F}$ & & $0.739 * *$ & $0.700 * *$ & $0.408^{* *}$ & $0.583 * *$ & $0.704 * *$ \\
\hline Curv $_{\mathrm{MF}}$ & & $0.692 * *$ & $0.666^{* *}$ & $0.380 * *$ & $0.571^{* *}$ & $0.657 * *$ \\
\hline $\mathrm{Eul}_{\mathrm{MF}}$ & & $0.675^{* *}$ & $0.670^{* *}$ & $0.429 * *$ & $0.663 * *$ & $0.673 * *$ \\
\hline
\end{tabular}

The lin./qua.fuzziness and log./exp.entropy in the neck is n.s. The highest values in each parameter group are rendered in italics n.s. not significant

${ }^{*} p<0.05 ; * * p<0.01$ 
Fig. 2 Total BMC versus FL, app. TbSp (head) versus FL/HD, f-BF (head) versus FL/HD, neck $m_{P(\alpha)}(\mathrm{SIM})$ versus FL/HD and $V_{\mathrm{MF}}$ versus FL. Solid lines display the regression curves
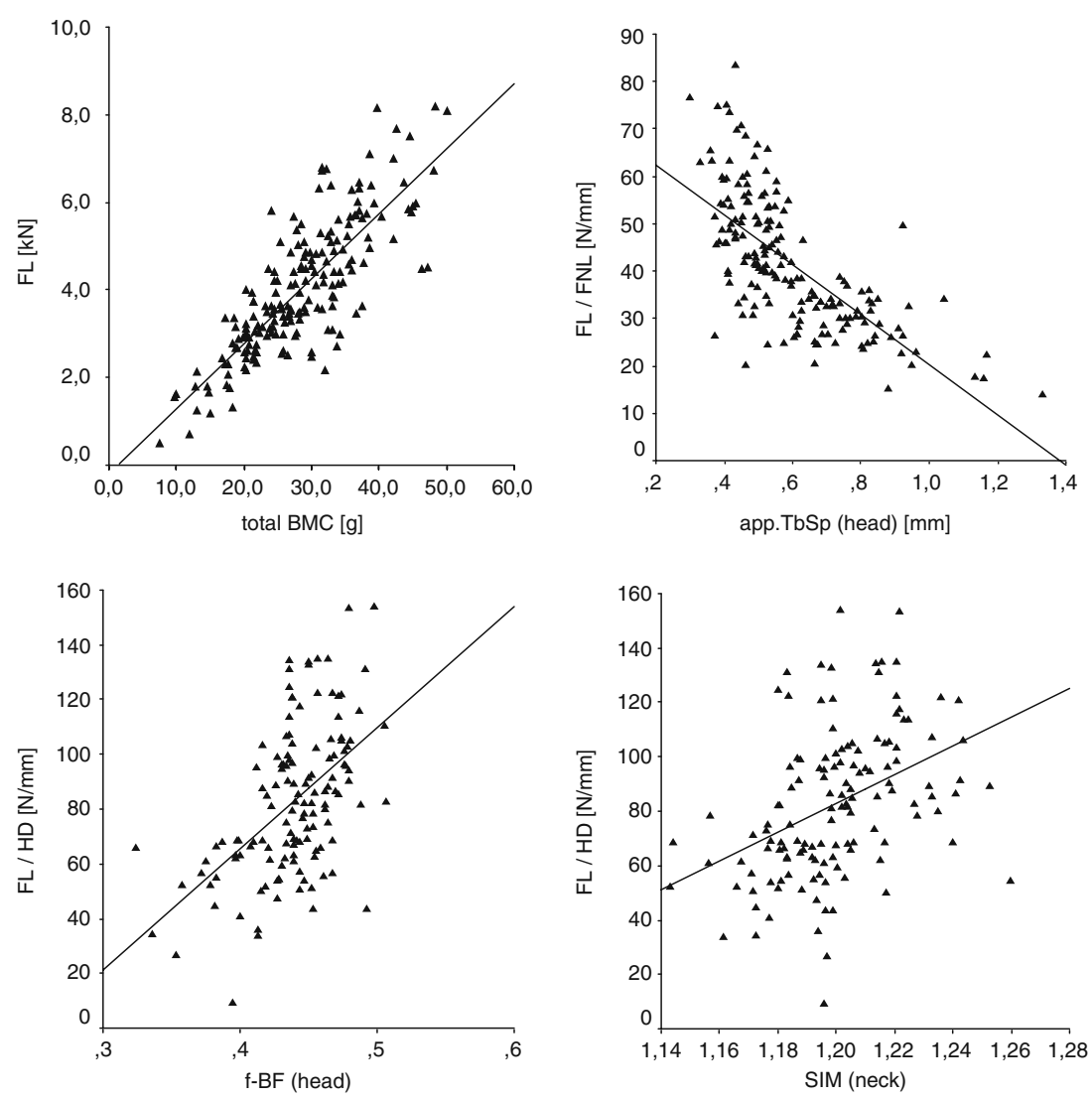

higher correlations were observed for BMD than for BMC. After adjustment of FL to BW, correlations of BMC, BMD, and all trabecular structure parameters remarkably decreased, suggesting a high adaptation of FL to BW. App. TbSp (morphometry) was the best single trabecular structure parameter predicting adjusted FL parameters, whereas the SIM and morphometry were the most notable trabecular structure parameters adding significant information in the linear regression models.

BMD achieved, in many cases, higher correlations with FL and adjusted FL parameters than trabecular structure parameters. This can be explained by the fact that DXA parameters comprehend not only information about the trabecular bone, but also about the cortical bone. It is well known that the cortical compartment contributes substantially to the mechanical properties of the bone [34]. Several studies reported significant correlations between cortical BMD, cortical structure parameters, and femoral bone strength [6, 35-37]. Trabecular structure parameters are not related to cortical bone properties, but can add significant information in combination with DXA in predicting femoral bone strength as shown in this study. They have complementary information to DXA and are potentially important for the assessment of femoral bone strength, even though they are not an integral whole-bone tool such as the finite element method [38-42]. 
Table 4 Best DXA and best multiple regression models for FL and adjusted FL parameters with corresponding $R_{\text {adj }}$

\begin{tabular}{lllll}
\hline & Best DXA & $R_{\text {adj }}$ & Best multiple & $R_{\text {adj }}$ \\
\hline FL & BMC (total) & 0.811 & BMC (total), exp.entropy (head), app.BF (trochanter), app.BF (head), $m_{P(\alpha)}($ head) & 0.840 \\
FL/BH & BMC (total) & 0.774 & BMC (total), Eul ${ }_{\mathrm{MF}}$, app.BF (trochanter), $m_{P(\alpha)}($ head), app.BF (head) & 0.819 \\
FL/BW & BMD (intertrochanteric) & 0.531 & BMD (intertrochanteric), app.TbN (head), app.TbTh (head) & 0.572 \\
FL/HD & BMD (neck) & 0.718 & BMD (neck), app.TbSp (head), f-BF (head), $m_{P(\alpha)}($ neck), app.TbN (neck) & 0.872 \\
FL/ND & BMD (neck) & 0.701 & BMD (neck), app.TbSp (head), f-BF (head), $m_{P(\alpha)}($ neck), app.TbN (neck) & 0.840 \\
FL/FNL & BMD (neck) & 0.757 & BMD (neck), $m_{P(\alpha)}($ head), Eul & 0.794 \\
FL/age & BMC (neck) & 0.735 & BMC (neck), Eul & 0.771 \\
\hline
\end{tabular}

DXA parameters had the highest correlations with FL in the neck ROI and the total ROI, similar to previous studies $[32,33]$. In contrast, trabecular structure parameters achieved the lowest correlations with FL and adjusted FL parameters mostly in the neck and the highest correlations by the majority in the femoral head. A direct comparison of DXA and trabecular structure parameters of the head was not possible, since DXA parameters were not measured in the femoral head due to the superimposition with the acetabulum in in vivo examination conditions.

To the best of our knowledge, we applied for the first time an automated 3D segmentation algorithm on CT images of the proximal femur for trabecular bone structure analysis. This algorithm has already been used for trabecular BMD analysis [24]. Several automated VOIfitting algorithms have been described for trabecular BMD analysis [6, 43], but none for trabecular bone structure analysis. Saparin et al. applied an automated 2D ROI placement on CT images of the femoral head and neck [44]. However, a 3D-based algorithm is essential to calculate 3D fuzzy logic, SIM, and MF and thus is advantageous. A limiting factor of the algorithm was the manual corrections of segmentation in 14 cases ( $7.5 \%$ of all specimens). These corrections can induce operator-dependent errors, but the determined reproducibility errors for segmentation indicated a good reproducibility of the morphometric parameters aside from app. TbSp in the neck. Reproducibility errors for segmentation and segmentation with repositioning were highest in the femur neck. Due to strong inhomogeneous bone structure in the femur neck, minor variations of the VOI position can induce major differences of the parameter values. Bauer et al. selected ROIs manually and reported highest reproducibility errors of the morphometric parameters also in the femur neck [13]. Reproducibility errors were considerably lower with our automated algorithm. They amounted to $0.11 \%$ to $9.41 \%$ for segmentation, compared to $1.8 \%$ to $31.3 \%$ using the manual technique of Bauer et al. This automated algorithm affords lower operator-dependent errors and additionally an enormous saving in time.
The calculation of the trabecular bone structure parameters has limitations. Images have to be binarized to compute the morphometric parameters and MF. Standardization was achieved by using the reference phantom, but the results are strongly dependent on the chosen threshold. No binarization is required for the application of fuzzy logic, but fuzzy logic parameters showed low correlations with FL and adjusted FL parameters. Concerning SIM, criteria for position and width of the two windows in the $P$ $(\alpha)$ spectrum are problematic. Standardized criteria are necessary and have to be determined in a later study. The shapes of the VOIs probably influence the structure analysis of the trabecular bone, since the proximal femur is very heterogeneous $[22,23]$. However, the chosen shapes of the VOIs in this study showed good reproducibility and were partly similar to ROIs used in previous studies [13, 14, 18]. Further limitations are the FL adjustment procedure and the precision error of the biomechanical test. The FL adjustment by division by BW, height, etc. may only in part capture the impact of these influencing variables. More complex adjustment procedures may offer additional insights into the performance of the various risk predictor variables tested. The error for the determination of FL in the biomechanical test is relatively high, approximately $15 \%$ based on a study of Eckstein et al. [28]. However, our analyses can be considered representative and statistically stable due to the large sample size $(n=187)$. Compared to our rather artificial in vitro setting, several challenges must be coped with in vivo. Error sources were reduced in this study, since CT and DXA acquisitions were not performed in situ. These impact the ability to extrapolate to the clinical setting and it remains to be investigated how the various parameters are affected. Segmentation of isolated bones is rather simple compared to in vivo segmentation and the effort is not comparable. Extraskeletal factors like neuromuscular diseases or vision disorders were not considered in this in vitro study, but are important to determine the risk of fracture [45].

In conclusion, an automated 3D segmentation algorithm was successfully applied to determine structure parameters 
of the trabecular bone using $\mathrm{CT}$ images of the proximal femur. The best single parameter predicting FL and adjusted FL parameters was app.TbSp (morphometry) or DXA-derived BMC or BMD. A combination of bone mass (DXA) and structure parameters of the trabecular bone (linear and nonlinear, global and local) most accurately predicted absolute and relative femoral bone strength.

Acknowledgements We thank the statistician, Petra Heinrich (Institut für Medizinische Statistik und Epidemiologie, Technische Universität München), for her advisory function in the statistical analysis, Simone Kohlmann, Volker Kuhn, and Maiko Matsuura for performing the biomechanical tests, as well as Holger Boehm, Simone Kohlmann, and Caecilia Wunderer for scanning the specimens. This work was supported by grants of the Deutsche Forschungsgemeinschaft (DFG LO 730/3-1 and MU 2288/2-2).

\section{Conflicts of interest None.}

Open Access This article is distributed under the terms of the Creative Commons Attribution Noncommercial License which permits any noncommercial use, distribution, and reproduction in any medium, provided the original author(s) and source are credited.

\section{References}

1. Boonen S, Autier P, Barette M, Vanderschueren D, Lips P, Haentjens P (2004) Functional outcome and quality of life following hip fracture in elderly women: a prospective controlled study. Osteoporos Int 15(2):87-94

2. Jiang HX, Majumdar SR, Dick DA, Moreau M, Raso J, Otto DD, Johnston DW (2005) Development and initial validation of a risk score for predicting in-hospital and 1-year mortality in patients with hip fractures. J Bone Miner Res 20(3):494-500

3. Damilakis J, Maris TG, Karantanas AH (2007) An update on the assessment of osteoporosis using radiologic techniques. Eur Radiol 17(6):1591-1602

4. Black DM, Greenspan SL, Ensrud KE, Palermo L, McGowan JA, Lang TF, Garnero P, Bouxsein ML, Bilezikian JP, Rosen CJ (2003) The effects of parathyroid hormone and alendronate alone or in combination in postmenopausal osteoporosis. N Engl J Med 349(13):1207-1215

5. Boehm HF, Eckstein F, Wunderer C, Kuhn V, Lochmueller EM, Schreiber K, Mueller D, Rummeny EJ, Link TM (2005) Improved performance of hip DXA using a novel region of interest in the upper part of the femoral neck: in vitro study using bone strength as a standard of reference. J Clin Densitom 8(4):488-494

6. Bousson V, Le Bras A, Roqueplan F, Kang Y, Mitton D, Kolta S, Bergot C, Skalli W, Vicaut E, Kalender W, Engelke K, Laredo JD (2006) Volumetric quantitative computed tomography of the proximal femur: relationships linking geometric and densitometric variables to bone strength. Role for compact bone. Osteoporos Int 17(6):855-864

7. Lang TF, Keyak JH, Heitz MW, Augat P, Lu Y, Mathur A, Genant HK (1997) Volumetric quantitative computed tomography of the proximal femur: precision and relation to bone strength. Bone 21 (1):101-108

8. Johnell O, Kanis JA, Oden A, Johansson H, De Laet C, Delmas P, Eisman JA, Fujiwara S, Kroger H, Mellstrom D, Meunier PJ, Melton LJ III, O'Neill T, Pols H, Reeve J, Silman A, Tenenhouse
A (2005) Predictive value of BMD for hip and other fractures. J Bone Miner Res 20(7):1185-1194

9. Schuit SC, van der Klift M, Weel AE, de Laet CE, Burger H, Seeman E, Hofman A, Uitterlinden AG, van Leeuwen JP, Pols HA (2004) Fracture incidence and association with bone mineral density in elderly men and women: the Rotterdam Study. Bone 34 (1):195-202

10. Carballido-Gamio J, Majumdar S (2006) Clinical utility of microarchitecture measurements of trabecular bone. Curr Osteoporos Rep 4(2):64-70

11. Link TM, Vieth V, Stehling C, Lotter A, Beer A, Newitt D, Majumdar S (2003) High-resolution MRI vs multislice spiral CT: which technique depicts the trabecular bone structure best? Eur Radiol 13(4):663-671

12. Phan CM, Matsuura M, Bauer JS, Dunn TC, Newitt D, Lochmueller EM, Eckstein F, Majumdar S, Link TM (2006) Trabecular bone structure of the calcaneus: comparison of MR imaging at 3.0 and $1.5 \mathrm{~T}$ with micro-CT as the standard of reference. Radiology 239(2):488-496

13. Bauer JS, Kohlmann S, Eckstein F, Mueller D, Lochmuller EM, Link TM (2006) Structural analysis of trabecular bone of the proximal femur using multislice computed tomography: a comparison with dual X-ray absorptiometry for predicting biomechanical strength in vitro. Calcif Tissue Int 78(2):78-89

14. Link TM, Vieth V, Langenberg R, Meier N, Lotter A, Newitt D, Majumdar S (2003) Structure analysis of high resolution magnetic resonance imaging of the proximal femur: in vitro correlation with biomechanical strength and BMD. Calcif Tissue Int 72(2):156-165

15. Wachter NJ, Augat P, Mentzel M, Sarkar MR, Krischak GD, Kinzl L, Claes LE (2001) Predictive value of bone mineral density and morphology determined by peripheral quantitative computed tomography for cancellous bone strength of the proximal femur. Bone 28(1):133-139

16. Boehm HF, Link TM, Monetti R, Kuhn V, Eckstein F, Raeth C, Reiser M (2006) Analysis of the topological properties of the proximal femur on a regional scale: evaluation of multi-detector CT-scans for the assessment of biomechanical strength using local Minkowski functionals in 3D. Proc SPIE 61446X.1:61446X.8

17. Boehm HF, Link TM, Monetti R, Mueller D, Rummeny EJ, Newitt D, Majumdar S, Raeth C (2004) Application of the Minkowski functionals in $3 \mathrm{D}$ to high-resolution MR images of trabecular bone: prediction of the biomechanical strength by nonlinear topological measures. Proc SPIE 5370:172-180

18. Boehm HF, Raeth C, Monetti RA, Mueller D, Newitt D, Majumdar S, Rummeny E, Morfill G, Link TM (2003) Local 3D scaling properties for the analysis of trabecular bone extracted from highresolution magnetic resonance imaging of human trabecular bone: comparison with bone mineral density in the prediction of biomechanical strength in vitro. Invest Radiol 38(5):269-280

19. Carballido-Gamio J, Phan C, Link TM, Majumdar S (2006) Characterization of trabecular bone structure from high-resolution magnetic resonance images using fuzzy logic. Magn Reson Imaging 24(8):1023-1029

20. Mueller D, Link TM, Monetti R, Bauer J, Boehm H, Seifert-Klauss V, Rummeny EJ, Morfill GE, Raeth C (2006) The 3D-based scaling index algorithm: a new structure measure to analyze trabecular bone architecture in high-resolution MR images in vivo. Osteoporos Int 17 (10):1483-1493

21. Patel PV, Eckstein F, Carballido-Gamio J, Phan C, Matsuura M, Lochmuller EM, Majumdar S, Link TM (2007) Fuzzy logic structure analysis of trabecular bone of the calcaneus to estimate proximal femur fracture load and discriminate subjects with and without vertebral fractures using high-resolution magnetic resonance imaging at $1.5 \mathrm{~T}$ and $3 \mathrm{~T}$. Calcif Tissue Int 81(4):294-304

22. Issever AS, Vieth V, Lotter A, Meier N, Laib A, Newitt D, Majumdar S, Link TM (2002) Local differences in the trabecular bone structure 
of the proximal femur depicted with high-spatial-resolution MR imaging and multisection CT. Acad Radiol 9(12):1395-1406

23. Nazarian A, Muller J, Zurakowski D, Muller R, Snyder BD (2007) Densitometric, morphometric and mechanical distributions in the human proximal femur. J Biomech 40(11):2573-2579

24. Huber MB, Carballido-Gamio J, Bauer JS, Baum T, Eckstein F, Lochmuller EM, Majumdar S, Link TM (2008) Proximal femur specimens: automated 3D trabecular bone mineral density analysis at multidetector $\mathrm{CT}$-correlation with biomechanical strength measurement. Radiology 247(2):472-481

25. Parfitt AM, Drezner MK, Glorieux FH, Kanis JA, Malluche H, Meunier PJ, Ott SM, Recker RR (1987) Bone histomorphometry: standardization of nomenclature, symbols, and units. Report of the ASBMR Histomorphometry Nomenclature Committee. J Bone Miner Res 2(6):595-610

26. Saha PK, Wehrli FW (2004) Measurement of trabecular bone thickness in the limited resolution regime of in vivo MRI by fuzzy distance transform. IEEE Trans Med Imaging 23(1):53-62

27. Michielsen K, de Raedt H (2001) Integral-geometry morphological image analysis. Phys Rep 347:461-583

28. Eckstein F, Wunderer C, Boehm H, Kuhn V, Priemel M, Link TM, Lochmuller EM (2004) Reproducibility and side differences of mechanical tests for determining the structural strength of the proximal femur. J Bone Miner Res 19(3):379-385

29. Gluer CC, Blake G, Lu Y, Blunt BA, Jergas M, Genant HK (1995) Accurate assessment of precision errors: how to measure the reproducibility of bone densitometry techniques. Osteoporos Int 5 (4):262-270

30. Wehrli FW, Gomberg BR, Saha PK, Song HK, Hwang SN, Snyder PJ (2001) Digital topological analysis of in vivo magnetic resonance microimages of trabecular bone reveals structural implications of osteoporosis. J Bone Miner Res 16(8):1520-1531

31. Wehrli FW, Ladinsky GA, Jones C, Benito M, Magland J, Vasilic B, Popescu AM, Zemel B, Cucchiara AJ, Wright AC, Song HK, Saha PK, Peachey H, Snyder PJ (2008) In vivo magnetic resonance detects rapid remodeling changes in the topology of the trabecular bone network after menopause and the protective effect of estradiol. J Bone Miner Res 23(5):730-740

32. Eckstein F, Lochmuller EM, Lill CA, Kuhn V, Schneider E, Delling G, Muller R (2002) Bone strength at clinically relevant sites displays substantial heterogeneity and is best predicted from site-specific bone densitometry. J Bone Miner Res 17(1):162-171

33. Lochmuller EM, Muller R, Kuhn V, Lill CA, Eckstein F (2003) Can novel clinical densitometric techniques replace or improve
DXA in predicting bone strength in osteoporosis at the hip and other skeletal sites? J Bone Miner Res 18(5):906-912

34. Augat P, Schorlemmer S (2006) The role of cortical bone and its microstructure in bone strength. Age Ageing 35(Suppl 2):ii27-ii31

35. Manske SL, Liu-Ambrose T, Cooper DM, Kontulainen S, Guy P, Forster BB, McKay HA (2008) Cortical and trabecular bone in the femoral neck both contribute to proximal femur failure load prediction. Osteoporos Int 20(3):445-453

36. Wachter NJ, Krischak GD, Mentzel M, Sarkar MR, Ebinger T, Kinzl L, Claes L, Augat P (2002) Correlation of bone mineral density with strength and microstructural parameters of cortical bone in vitro. Bone 31(1):90-95

37. Manske SL, Liu-Ambrose T, de Bakker PM, Liu D, Kontulainen S, Guy P, Oxland TR, McKay HA (2006) Femoral neck cortical geometry measured with magnetic resonance imaging is associated with proximal femur strength. Osteoporos Int 17(10):1539-1545

38. Bessho M, Ohnishi I, Okazaki H, Sato W, Kominami H, Matsunaga S, Nakamura K (2004) Prediction of the strength and fracture location of the femoral neck by CT-based finite-element method: a preliminary study on patients with hip fracture. J Orthop Sci 9(6):545-550

39. Bessho M, Ohnishi I, Matsuyama J, Matsumoto T, Imai K, Nakamura K (2007) Prediction of strength and strain of the proximal femur by a CT-based finite element method. J Biomech 40(8): $1745-1753$

40. Keyak JH, Falkinstein Y (2003) Comparison of in situ and in vitro CT scan-based finite element model predictions of proximal femoral fracture load. Med Eng Phys 25(9):781-787

41. Yosibash Z, Trabelsi N, Milgrom C (2007) Reliable simulations of the human proximal femur by high-order finite element analysis validated by experimental observations. J Biomech 40(16):36883699

42. Yosibash Z, Padan R, Joskowicz L, Milgrom C (2007) A CT-based high-order finite element analysis of the human proximal femur compared to in-vitro experiments. J Biomech Eng 129(3):297-309

43. Li W, Sode M, Saeed I, Lang T (2006) Automated registration of hip and spine for longitudinal QCT studies: integration with 3D densitometric and structural analysis. Bone 38(2):273-279

44. Saparin P, Thomsen JS, Kurths J, Beller G, Gowin W (2006) Segmentation of bone CT images and assessment of bone structure using measures of complexity. Med Phys 33(10):3857-3873

45. Dontas IA, Yiannakopoulos CK (2007) Risk factors and prevention of osteoporosis-related fractures. J Musculoskelet Neuronal Interact 7(3):268-272 